\title{
Surface Acoustic Streaming in Microfluidic System for Rapid multicellular Tumor Spheroids generation
}

\author{
Layla AlHasan ${ }^{1,2}$, Aisha Qi ${ }^{2,}$ Aswan Al-Aboodi ${ }^{3,}$ Amged Rezk ${ }^{1,}$ Richie R. Shilton ${ }^{1,}$ Peggy P.Y. \\ Chan $^{1,4}$, James Friend ${ }^{1,4}$ and Leslie Yeo ${ }^{1}$ \\ ${ }^{1}$ Micro/Nanophysics Research Laboratory, RMIT University, Melbourne, VIC 3000, Australia. \\ ${ }^{2}$ Biotechnology and Biological sciences, School of Applied Sciences, RMIT University, Melbourne, VIC 3000, \\ Australia. \\ ${ }^{3}$ Department of Chemical Engineering, Monash University, Clayton, VIC 3800, Australia. \\ ${ }^{4}$ Melbourne Centre for Nanofabrication (MCN), Clayton, VIC 3000, Australia
}

\begin{abstract}
In this study, we developed a novel and rapid method to generate in vitro three-dimensional (3D) multicellular tumor spheroids using a surface acoustic wave (SAW) device. A SAW device with single-phase unidirectional transducer electrodes (SPUTD) on lithium niobate substrate was fabricated using standing UV photolithography and wet-etching techniques. To generate spheroids, the SAW device was loaded with medium containing human breast carcinoma (BT474) cells, an oscillating electrical signal at resonant frequency was supplied to the SPUDT to generate acoustic radiation in the medium. Spheroids with uniform size and shape can be obtained using this method in less than 1 minute, and the size of the spheroids can be controlled through adjusting the seeding density. The resulting spheroids were used for further cultivation and were monitored using an optical microscope in real time. The viability and actin organization of the spheroids were assessed using live/dead viability staining and actin cytoskeleton staining, respectively. Compared to spheroids generated using the liquid overlay method, the SAW generated spheroids exhibited higher circularity and higher viability. The F-actin filaments of spheroids appear to aggregate compared to that of untreated cells, indicating that mature spheroids can be obtained using this method. This spheroid generating method can be useful for a variety of biological studies and clinical applications.
\end{abstract}

\section{INTRODUCTION}

Monolayer cultures are commonly used in cancer research and anti-tumour drug development. However, monolayer cultures suffered the loss of stroma components and extracellular matrix, and therefore cannot reflect in vivo microenvironment realistically. Three-dimensional (3D) multicellular spheroids are frequently used in cancer and morphogenesis studies, as 3D spheroids can mimic the microenvironment of in vivo tissues more realistically compared to monolayer culture $(1,2)$. Different cell types such as hepatocytes (3) and embryonic stem cells $(2,3)$ can form 3D spheroids in vitro. For example, liver hepatocellular carcinoma cells (HepG2) has been used to form 3D spheroids, these spheroids can maintain their viability and functions as well as expressing 500 more protein markers compared to monolayer culture (4). Liquid overlay and hanging drop methods are commonly being used to generate 3D spheroids; however, these methods involved laborious and tedious procedures (5).

Herein, we developed a surface acoustic wave (SAW) microfluidic device and developed a simple protocol to produce in vitro 3D tumor spheroids with uniform size within a minute. The SAW device has shown to rotate and move fluid with micro-volumes in a rapid and controllable fashion through surface acoustic streaming. The SAW device is small, disposable and low cost, and is therefore advantageous for drug delivery applications (6). In this study, we generated BT474 breast cancer cell spheroids using the SAW device and evaluate the physiochemical properties of these spheroids.

Micro/Nano Materials, Devices, and Systems, edited by James Friend, H. Hoe Tan, Proc. of SPIE Vol. 8923, 89235C - (C) 2013 SPIE - CCC code: 0277-786X/13/\$18 - doi: 10.1117/12.2034050 


\section{METHODOLOGY}

To generate spheroids, the SAW device was loaded with medium containing human breast carcinoma (BT474) cells, an oscillating electrical signal at resonant frequency was supplied to the SPUDT to generate surface acoustic streaming in the medium. Spheroids with uniform size and shape can be obtained using this method in less than 1 minute as shown in Fig.1.

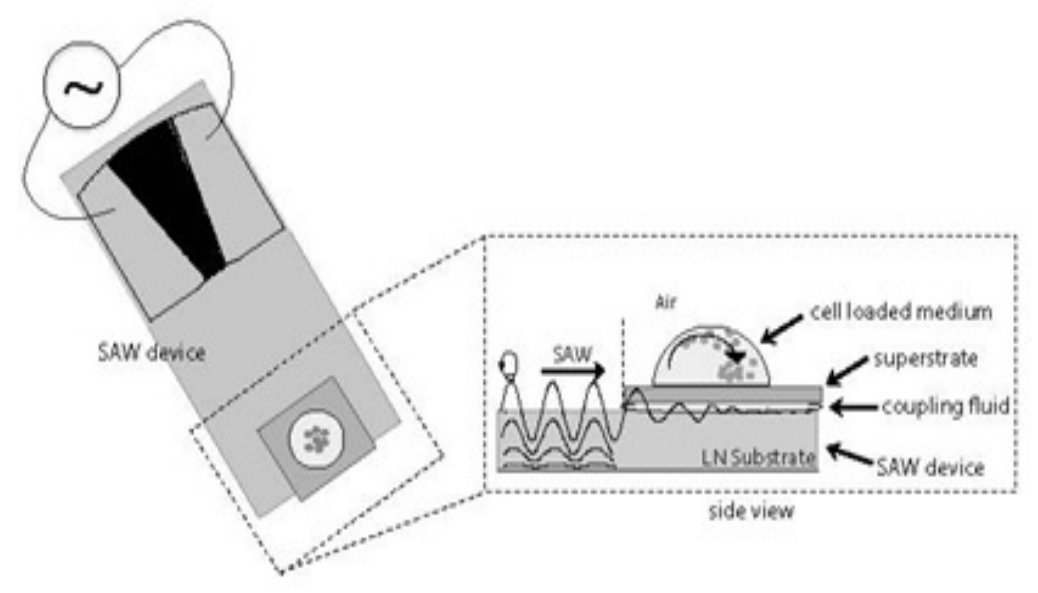

Fig.1: Spheroids generation by Surface Acoustic Wave (SAW). Not to scale.

We compared tumor spheroids generated by the SAW device to those generated by conventional liquid overlay method. The spheroids morphology was evaluated using optical microscopy, the spheroid sizes were measured using image processing software ImageJ. The viability of the spheroids was investigated according to the procedure described in (7) using a LIVE/DEAD staining kit (Life Technologies Pty Ltd, Australia), these spheroids were further examined using actin filament staining. All experiments were repeated three times.

\section{RESULTS}

BT474 spheroid can be generated by the SAW device within one minute. At day 1, the SAW generated spheroids exhibited spherical morphology as shown in representative confocal microscopy image (Fig. 2A). In contrast, spheroid generated using the same number of cells by conventional liquid overlay method exhibited irregular shape starting from the first day. After 7 days of cultivation the liquid overlay method generated spheroids continued to exhibit irregular morphology, whereas SAW generated spheroids continued to exhibit spherical morphology.

The cell viability of tumor spheroids formed by the SAW streaming and the liquid overlay method was examined. Viable cells can be stained by Calcein AM and appeared in green. It can be seen in Fig. 2 that most cells in the periphery layer of spheroids (SAW generated and control) remained viable, where cells at the centre of the spheroids lost their viability due to lack of oxygen and nutrients exchange, such necrotic core feature is commonly being observed in large spheroid (8). Factin filaments of SAW generated spheroids appear to aggregate compared to that of monolayer cells (data not shown), indicating that mature spheroids can be obtained using this method. 


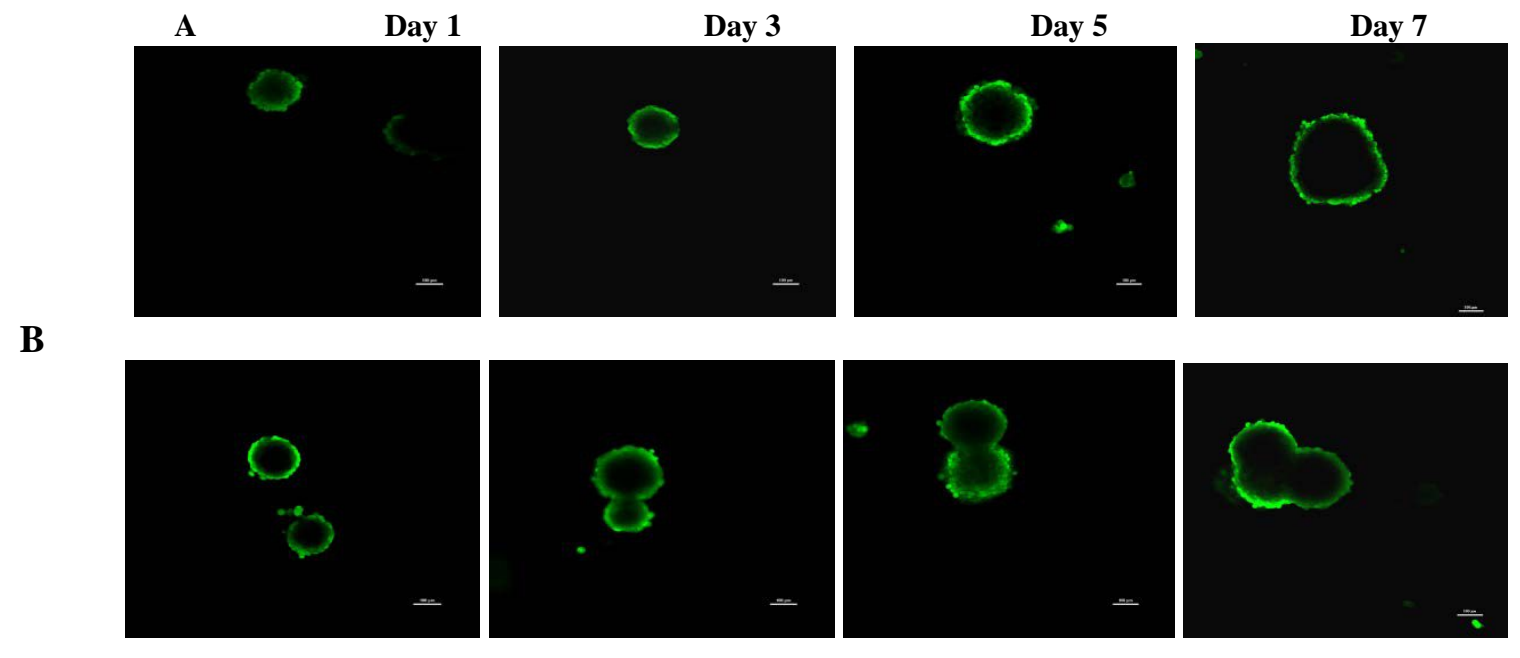

Fig.2: Generation of tumor spheroids by (A) the SAW device and (B) liquid overlay method. Spheroids are stained with Live/ Dead kit; live cells are stained in green. Scale bar represents $100 \mu \mathrm{m}$.

\section{CONCLUSION}

A surface acoustic wave device is developed for rapid 3D spheroid generation. Compared to conventional liquid overlay method, SAW method can generate spheroid efficiently within 1 min using simple approach. The resulting spheroids were found to be viable and exhibited uniform in morphology. The spheroids produced by this method can be useful for various drug-screening, cancer, and tissue morphogenesis studies.

\section{REFERENCES}

[1] Torisawa YS, Mosadegh B, Luker GD, Morell M, O'Shea KS, Takayama S. Microfluidic hydrodynamic cellular patterning for systematic formation of co-culture spheroids. Integrative biology: quantitative biosciences from nano to macro. 2009 1(11-12):649-54.

[2] Jeong GS, Jun Y, Song JH, Shin SH, Lee SH. Meniscus induced self-organization of multiple deep concave wells in a microchannel for embryoid bodies generation. Lab on a chip. 2012; 12(1):159-66.

[3] El-Ali J, Sorger PK, Jensen KF. Cells on chips. Nature. 2006; 442(7101):403-11.

[4]. Ota H NM. Microfluidic experimental platform for producing size-controlled three-dimensional spheroids. Sensors and Actuators A. 2011; 169:266-73.

[5] Arbatan T, Al-Abboodi A, Sarvi F. Chan PPY, Shen W. Tumor inside a pearl drop, Advanced Healthcare Materials. 2012; 1:467-469.

[6] Yeo LY, Chang HC, Chan PP, Friend JR. Microfluidic devices for bioapplications. Small. 2011; 7(1):12-48. 
[7] Lim TC, Toh WS, Wang LS, Kurisawa M, Spector M. The effect of injectable gelatinhydroxyphenylpropionic acid hydrogel matrices on the proliferation, migration, differentiation and oxidative stress resistance of adult neural stem cells. Biomaterials. 2012; 33(12):3446-55.

[2] Hamilton G, Multicellular spheroids as an in vitro tumor model, Cancer Letters. 1998; 131(1): 29-34. 